\title{
Elenco delle abbreviazioni
}

$\mathrm{ACTH}$

AGT

AMP, ciclico

CRH, CRF

DEAS

DL 50

DNA

DOCA

$\mathrm{EE}$

FSH

FSH-RF

FSH-RH, FRH, FRF GH-RH, GRH, GRF GIH, GIF

Gn-RH ( = LH-RH e FSH-RH)

GT

$\mathrm{HCG}$

HMG

HPL (= HCS)

ICSH ( = LH)

ICSH-RF

LH ( = ICSH)

LH-RH, LH-RF

LTH (= LGH = PRL)

MAO

MCR

ME ormone adrenocorticotropo $=$ corticotropina

attività gonadotropa totale

adenosinmonofosfato

ormone che libera la corticotropina

deidroepiandrosterone solfato

dose letale per il $50 \%$ degli animali

acido desossiribonucleico

desossicorticosterone acetato

etinilestradiolo

ormone follicolostimolante

fattore che libera l'ormone follicolostimolante, releasing factor per l'FSH

ormone che libera l'ormone follicolostimolante

ormone che libera l'ormone della crescita

somatostatina $=$ ormone inibente la liberazione dell'or-

mone della crescita

ormone che libera le gonadotropine

gonadotropine

gonadotropina umana corionica

gonadotropina umana menopausale

human chorionic lactogen $=$ lattogeno placentare $=$ hu-

man placental lactogen $=$ human chorionic somatomammotrophin

ormone stimolante le cellule interstiziali

ICSH releasing factor

ormone luteinizzante

ormone che libera l'ormone luteinizzante, releasing factor per l'LH

prolattina $(=\mathrm{HPr})=$ ormone luteotropo $=$ luteotropina $=$ ormone lattogeno $=$ galattina $=$ mammotropina

monoaminoossidasi

metabolic clearance rate $=$ tasso di clearance metabolica

mestranolo 
MRIH, MRIF, MIF ormone inibente la liberazione dell'ormone melanocitostimolante

MSH-RH, MRH, MRF ormone che libera l'ormone melanocitostimolante

ng

pg nanogrammo

PG

$\mathrm{PG} \mathrm{E}_{2}$

$\left.P G F_{2} \alpha\right\}$

picogrammo

prostaglandine

PIF, PRIH

denominazione di alcune prostaglandine

PMS

ormone inibente la liberazione di prolattina

pregnant mare serum = gonadotropina serica di giumenta gravida

PRH, PRF

ormone che libera la prolattina

$\mathrm{PU}=\mathrm{HCG}$

pregnancy urine hormone

RIA

RNA

radio-immuno-assay, dosaggio radioimmunologico

SGOT

SGPT

SNC

acido ribonucleico

transaminasi serica glutammico-ossalacetica

transaminasi serica glutammico-piruvica

sistema nervoso centrale

STH

TB

ormone somatotropo $=$ ormone della crescita

temperatura basale

TSH ( = TTH)

ormone tireotropo = tireotropina

TS-RH, TRH, TRF

$\mathrm{U}$

ormone che libera la tireotropina

unità

UI

unità internazionali

UIB

unità benzoica internazionale 